evident at any time and as the capacity of the suspensory ligament to transmit excess fluid is very limited, I incline to the view that the sub-retinal fluid was, in part at least, derived from the vitreous humour. The vitreous colloids being saturated with water are isoelectric with their surroundings and exert no colloid or onkotic pull, but an active inflammatory exudate, derived from the uveal vessels and situated between the cerebral and epithelial layers of the retina, would exert a decided pull upon the vitreous fluid, especially if the inner layer of the retina were damaged by inflammatory or traumatic insult. As the conditions present are compatible with displacement of fluid from the vitreous chamber either through the suspensory ligament or through the inner layer of the retina, further study will be necessary to enable an intelligent estimate of the degree in which each of these factors is active, to be formed.

\title{
NOTE ON CONCRETIONS IN THE LACRYMAL CANALICULI
}

\author{
BY
}

R. R. JAMES

LONDON

THOUGH concretions in the canaliculi are not very frequently seen, they are perfectly well known to all ophthalmic surgeons as a cause of intractable conjunctivitis. I recently had such a case in a young man where the concretion was lodged in the upper canaliculus. The lower canaliculus was quite free and there was no interference with the passage of fluid on syringing the tear sac. It was not until a purulent secretion was observed flowing from the upper punctum on pressure over the inner canthus that the correct diagnosis was made. On slitting up the upper canaliculus the fragments of the concretion were easily expressed. The improvement was immediate, the patient announcing, when I saw him a fortnight later, that for the first time for 18 months the eye had been free from discharge and stickiness. The débris examined by the pathologist showed a profuse streptothrix growth.

It is easy to understand how, in the pressure of out-patient ophthalmic work, such a case can be overlooked. The conjunctival condition improves up to a point with the usual local treatment, but never gets quite well, and it is easy to see why. I would plead that in such cases the surgeon should not be content with investigating the state of the lower canaliculus only : he must remember that the upper canaliculus may be, after all, the site of the trouble. 